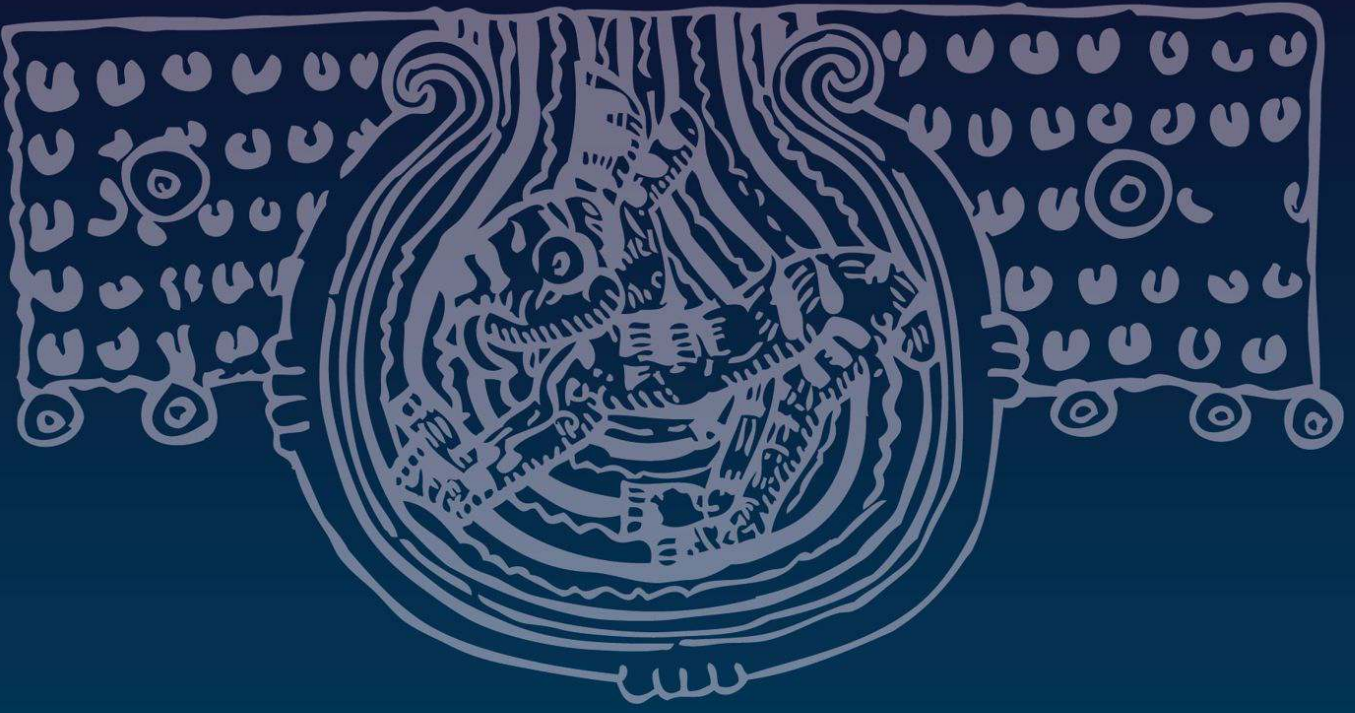

Las cosas de la

NOCHE

UNA MiRAdA difERENTE

Aurore Monod Becouelin JaCOUES Galinier COORdinadores

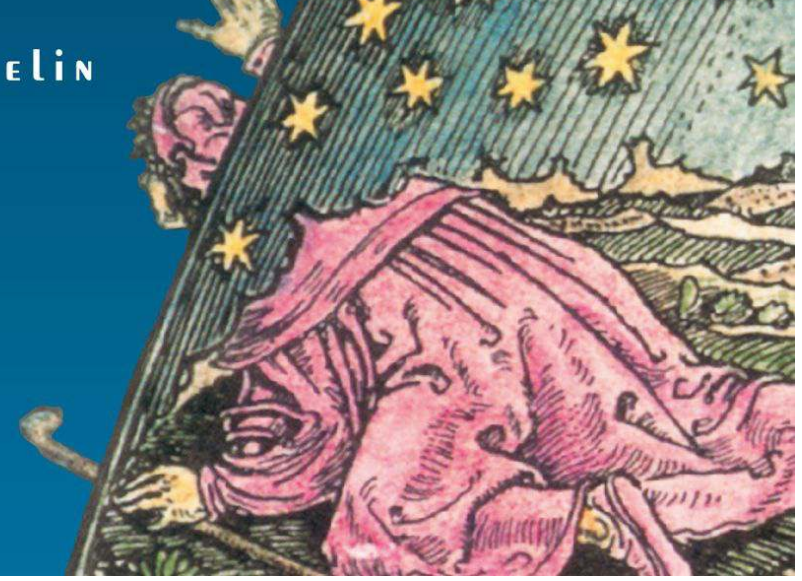




\section{Las cosas de la noche}

Una mirada diferente

\section{Aurore Monod Becquelin y Jacques Galinier (dir.)}

Editor: Centro de estudios mexicanos y

centroamericanos

Lugar de edición: México

Año de edición: 2016

Publicación en OpenEdition Books: 6

décembre 2016

Colección: Antropología y Etnología

\section{Sbooks}

http://books.openedition.org

\section{Referencia electrónica}

MONOD BECQUELIN, Aurore (dir.) ; GALINIER, Jacques (dir.). Las cosas de la noche: Una mirada

diferente. Nueva edición [en línea]. México: Centro de estudios mexicanos y centroamericanos, 2016 (generado el 07 diciembre 2016). Disponible en Internet: <http://books.openedition.org/cemca/4201>

Este documento fue generado automáticamente el 7 décembre 2016.

(c) Centro de estudios mexicanos y centroamericanos, 2016

Condiciones de uso:

http://www.openedition.org/6540 


\title{
La Noche, nuevo objeto de salvaguarda
}

\author{
Samuel Challéat
}

Tradución : Mario Zamudio Vega

1 Reserva Internacional de Cielo Estrellado: este nombre, un recién llegado en la larga lista de los medios de reserva y protección de los "objetos naturales", suena como un enésimo medio de defensa de un objeto sectorial específico (el "cielo estrellado" de una categoría de actores: los astrónomos), en resumen, un enésimo medio -institucionalizado, sin duda alguna, y hecho al gusto medioambiental del día- de defensa de un patrimonio. Ha sido lanzado el término Reserva, que nos remite al perímetro, a la delimitación, a la frontera y, por ende, a la protección, e incluso a la sacralización. Entonces, para proteger el cielo estrellado, ¿"salvemos la noche"?; pero, ¿de qué y, por lo tanto, de quién es el ¡no! a este llamado? ¿Frente a qué enemigo que invade a la comunidad internacional debe ésta levantar incluso fronteras de reservas? La respuesta podría sorprender, incluso consternar, y no dejar a nadie indiferente mientras ese invasor ocupe legítimamente el lugar, en la conciencia colectiva, de objeto de progreso. Porque es claro que se trata de un "enemigo íntimo", puesto que es utilizado por todos en los países industrializados; je incluso más!: se trata de un objeto del que cada uno de nosotros -y, a fortiori, nuestras sociedades- se ha vuelto dependiente: la luz artificial (Challéat, 2010).

Por consiguiente, ha sido necesario un cambio radical de la percepción misma de la luz artificial, haciéndola pasar de su condición cuasi exclusiva de objeto de progreso a la de un objeto que también puede ser perjudicial. Por supuesto, ese deslizamiento -todavía actuante en el presente- no se produjo en un día y, como ocurre con mucha frecuencia en materia de urgencia en la conciencia colectiva de un nuevo objeto de riesgos o daños al medio ambiente, el proceso ha generado previamente movimientos de personas directamente afectadas -en sus actividades y debido a su lugar de residencia- por ese objeto. ${ }^{2}$ De la molestia experimentada al perjuicio y, después, del perjuicio a la contaminación reconocida científicamente, la gradación en la expresión del daño y, luego, en su aceptación científica y política (la cual lleva incluso al establecimiento de reservas, medios de protección, etcétera) es grande y, sobre todo, no cae por su propio peso. De 
este modo se constituye todo objeto perjudicial, así como la urgencia de ubicarlo en un contexto particular: histórico, social, económico, político, etcétera.

\section{El surgimiento de la luz artificial como objeto de perjuicio}

Lucifer ("que porta la luz", etimológicamente, del latín lux, 'luz', y fero, 'portar') fue expulsado del cenit al nadir y se convirtió en Satanás, el príncipe de las Tinieblas y espíritu del Mal, opuesto a la Luz creada por el Verbo, la Palabra de Dios. Dualidad, naturaleza doble antitética que evoca las oposiciones y, asimismo -jineluctablemente! -, las complementariedades: como en el caso del ritmo en dos tiempos de la vida y la muerte, del pasado y el porvenir, del blanco y el negro, de la luz y la obscuridad, del día y la noche. Esta última, debido a que es el tiempo de la sombra, de las tinieblas-y, por ende, de los temores-, sigue estando cargada, todavía hoy en día, por una mitología que la asocia al mal, el complot y la inseguridad; ahora bien, a pesar de ello, la noche no ha dejado de ser, para muchos, el momento de los placeres, de la fiesta y de la transgresión de ciertas "prohibiciones sociales". En consecuencia, ya no es necesario recordar a qué punto nuestra llamada sociedad "desarrollada" sostiene una relación compleja con la noche, ese espacio-tiempo fuera de la cadencia diurna que favorece la reflexión, la imaginación, la escucha, la creación, el acercamiento con el otro, al mismo tiempo que revela la segregación, el temor y, por lo tanto, la limitación y la restricción.

Por consiguiente, es necesario hablar de una noche construida -dado que es social- en los límites muy diferenciados de los simples mojones astronómicos que la hacen correr precisamente de la puesta a la salida del Sol. El tiempo nocturno, ya sea el de la fiesta, el del trabajo o el del reposo, no es la simple prolongación del tiempo diurno, sino que constituye claramente un universo en sí, que surge históricamente como objeto por derecho propio por medio de su conquista en y por la ciudad. En consecuencia, en los siglos XVII y XVIII, es, ante todo, un tiempo sobre el cual el orden urbano tiene dificultades para imponerse (Cabantous, 2009). Posteriormente, es un espacio-tiempo que debe humanizarse y racionalizarse para permitir, en el transcurso del siglo xIx, el nacimiento del noctambulismo, modo de ser en la ciudad revelador de una nueva sensibilidad citadina asociada con una nueva relación con el tiempo y el espacio vial (Delattre, 2004). Por medio de su percepción del tiempo largo, el historiador pone de relieve el proceso de colonización de la noche occidental -tanto de la urbana como de la rural- en nombre de lo que se puede llamar un "proyecto luz" intrínsicamente vinculado posteriormente al proyecto de las Luces (Koslovsky, 2011). 


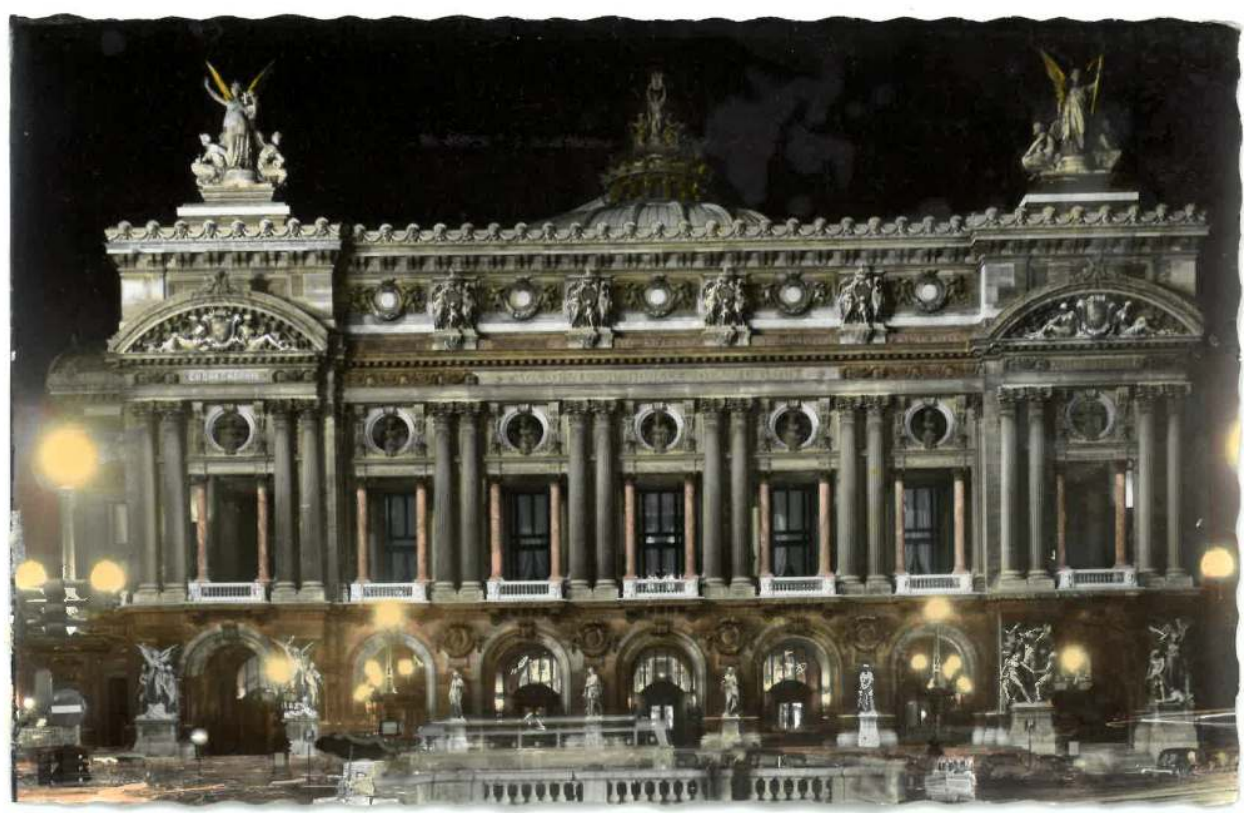

"Paris la nuit, l'Opéra”. Principios del siglo Xx. Fotografía extraída de la serie "Paris la nuit - 10 photos véritables en couleur, Édition E.R., Paris, Véritable photo au bromure". Archivo del Colectivo RENOIR.

Esteticismo urbano 2.

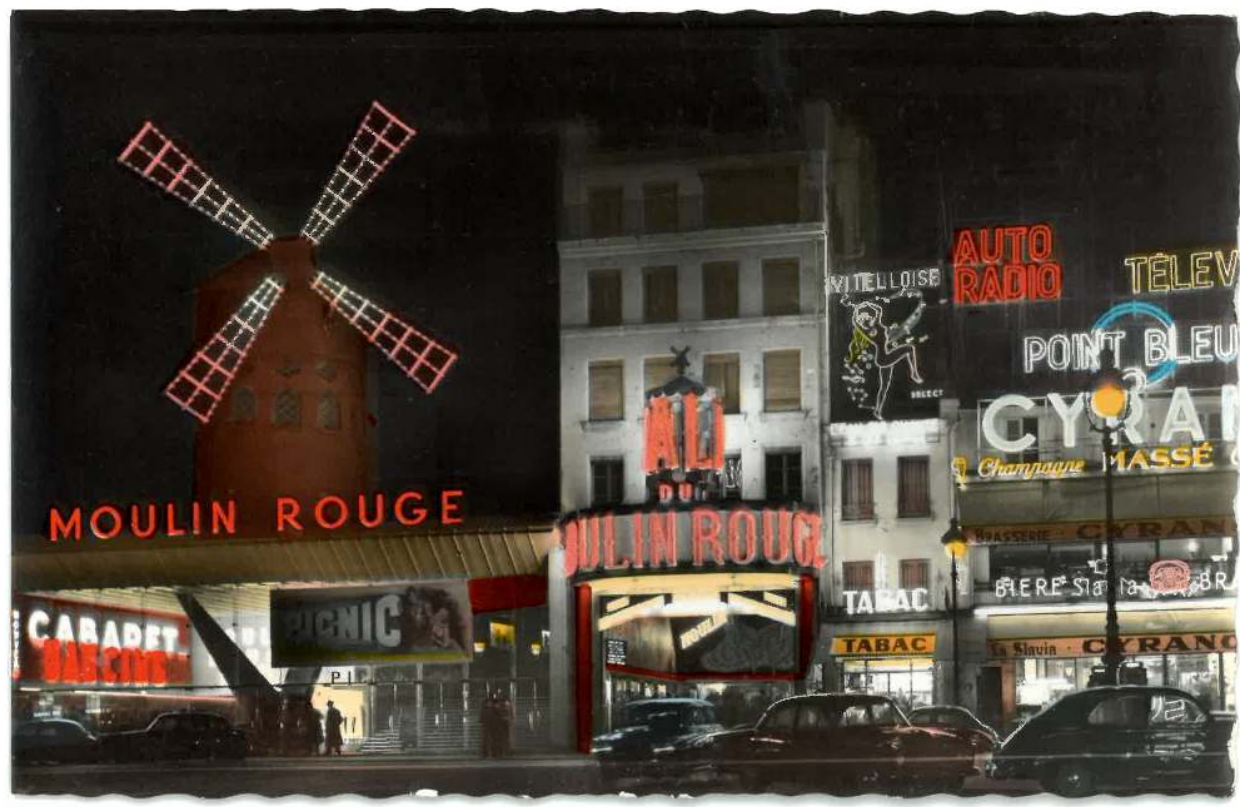

"Paris la nuit, Le Moulin Rouge". Principios del siglo XX. Fotografía extraída de la serie "Paris la nuit 10 photos véritables en couleur, Édition E.R., Paris, Véritable photo au bromure". Archivo del Colectivo RENOIR.

5 La concepción del alumbrado urbano, verdadera constante histórica, se ha basado marcadamente en la voluntad de responder a los temores sociales por medio de la sumisión de la calle al orden. Todavía en la actualidad, la voluntad de visibilidad a giorno 
de las instituciones se opone a lo nocturno, percibido por el político como hacedor de la carencia de derecho: la propia transgresión que permite a algunos la liberación no está lejos de ser la causa, para los otros, de la amenaza y la inseguridad, atavismo perpetuo asociado con la noche y, con mayor motivo, cuando se trata de la periferia urbana; no obstante, en la actualidad se atribuye otras funciones al alumbrado artificial, el que, desde los años de 1980, debe -además de garantizar la seguridad de los lugares, los bienes, las personas y los desplazamientos por la ciudad- formar parte de la recomposición de los espacios y de la promoción de lo urbano por medio de lo que se ha convenido en llamar "la iluminación". Así, en los documentos sobre urbanismo, han visto la luz varios instrumentos para proporcionar una escenografía de la luz que con mucha frecuencia forma parte de la tendencia de la ciudad a su "dedicación al turismo (Mons, 2000: 201).

6 Por lo demás, recientemente se han alzado ciertas voces -incluso en el seno de la joven profesión de los diseñadores de la iluminación- con el propósito de prevenir sobre el riesgo de un exceso de la iluminación, que podría formar parte de la fabricación de un centro de la ciudad convertido en museo, el que, en ocasiones, tiene el aspecto nocturno de un parque de atracciones; pero, más objetivamente, lo que puede revelarse negativo son los diferentes efectos de la luz artificial nocturna sobre la visión del cielo estrellado como recurso científico y cultural y sobre el medio ambiente nocturno, así como sobre la salud humana.

7 Las primeras expresiones de la puesta en tela de juicio de la luz artificial surgieron en los años de 1970 en Estados Unidos y, después, en Europa Occidental. Fue un periodo de extensión urbana generalizada durante el cual, en consecuencia, bajo los efectos del desplazamiento de automóviles cada vez más veloces, tuvo lugar la colonización que llevaron a cabo las ciudades, de los espacios que hasta entonces eran predominante rurales con el propósito de responder a un nuevo modo de habitar la ciudad, el de la casa individual tipo chalet; pero, asimismo, fue un lapso extremadamente complejo que generó mutaciones de la infraestructura de producción de electricidad $\mathrm{y}$, por consiguiente, de la infraestructura de distribución. En ese sentido, Francia es emblemática: es un país que, durante ese tiempo, adoptó la decisión de recurrir de manera generalizada a la energía eléctrica nuclear, con centrales que sólo pueden funcionar al máximo, una energía que la empresa Électricité de France (EDF) incitaría a consumir -por medio de tarifas atractivas para las colectividades territoriales-, antes bien que perderla. De manera subsecuente a su aumento a la medida, las ciudades y los pueblos vieron cómo se densificaba e intensificaba su parque de alumbrado público, lo cual hizo crecer los domos de luz artificial formados sobre ellos.

8 Esos halos luminosos impiden el acceso al cielo estrellado a algunos individuos -poco numerosos, hay que señalarlo-, astrónomos profesionales y aficionados que, con el propósito de fundamentar su puesta en tela de juicio del objeto luz artificial, construirían y reivindicarían un nuevo bien medioambiental por derecho propio y con una fuerte carga simbólica: el cielo estrellado. Con todo, el hecho de que algunos individuos expresaran su molestia, a pesar de que estaba en concordancia con el contexto del pensamiento medioambiental favorable de los años de 1970 no fue suficiente, aún faltaría mucho para que se relegara la luz artificial a la categoría de elemento contaminante. Sería necesario esperar hasta los años 1990 y, más todavía, hasta los primeros años de este siglo, después de que los ecólogos y, más tarde, los médicos hubiesen adoptado la temática, para que la luz artificial fuese percibida científicamente como un verdadero "alterógeno" de la negrura de la noche, estado físico que, de manera natural, forma parte 
integral de todo biotopo y una necesidad, desde el punto de vista de la calidad, del medio ambiente saludable: para los primeros, la luz artificial constituye una nueva causa de erosión generalizada de la biodiversidad; para los segundos, está a punto de adquirir la condición de perturbador endócrino y, por ende, de factor que facilita riesgos sanitarios.

En consecuencia, desde hace unos veinte años ha surgido un nuevo campo de saber científico que permite pensar conceptualmente a la luz artificial como contaminante $y$, por consiguiente, de manera recíproca, en la noche como un objeto que se debe salvaguardar. Por lo tanto, las reivindicaciones de los actores históricos se tornaron en lo sucesivo hacia la salvaguarda, ya no únicamente del cielo negro estrellado, sino también de la noche negra en su totalidad, menos sectorial $\mathrm{y}$, por ende, más federativa. Este aumento de generalidad, alimentado por el saber científico ecológico y médico, les permitió en lo sucesivo exigir que se tomara en consideración la problemática de la iluminación artificial en los textos de referencia de las acciones públicas y privadas. Como consecuencia, el objeto del perjuicio pasó de la condición de una molestia reivindicada de manera sectorial a la de un daño y, después, a la de una contaminación cuyo tratamiento se encuentra en lo sucesivo incluido en la agenda política.

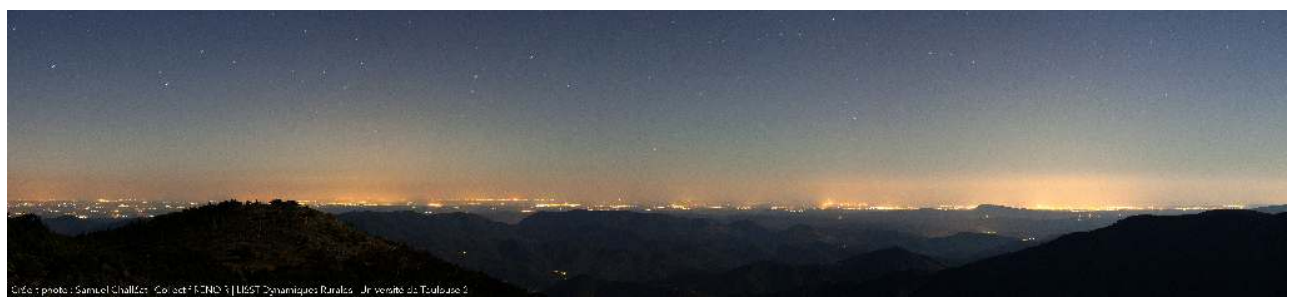

HALOS LUMINOSOS FORMAdOS POR ENCIMA DE LAS AgLOMERACIONES DE AVIÑóN, NIMES Y MONTPELLIER (DE IZQUIERDA A DERECHA). VISTA TOMADA DESDE EL MONTE AIgOUAL (1 567 METROS De ALtURA, PARQUE NACIONAL de LAS CEVENAS, FRANCIA).

FOTOgRAFÍA: SAMUEL CHALLÉAT.

\section{Los conflictos surgidos de los usos contradictorios de lo nocturno}

10 A la gradación de la expresión del perjuicio corresponde la gradación de las reivindicaciones de las llamadas asociaciones de "defensa de lo nocturno". Desde luego, su deseo de ver que se disminuyera la cantidad de luz artificial emitida por las ciudades no fue recibida favorablemente por los actores de la producción de luz artificial (fabricantes de luminarias, instaladores, diseñadores de la iluminación, profesionales y sindicatos de la energía, etcétera), para los que se trata de una profunda puesta en tela de juicio de su competencia profesional. En consecuencia, la conflictividad se instaló rápidamente entre esos actores, conflictividad que se expresaba, ante todo, entre los actores de las asociaciones, promotores de reivindicaciones, según una lógica a la Hirschman de la voz (Hirschman, 1970); y se inició una verdadera lucha en contra del alumbrado público urbano, lucha que se puede comparar con una "lucha de los lugares" en contra de la "urbanización rampante". Muy pronto, por lo tanto, se hicieron públicos desde los primeros rasgos de sensibilización, las primicias de la idea de reservas localizadas del cielo nocturno frente a la urbanización.

11 Posteriormente, muy a la manera clásica en materia de conflictos medioambientales, las interacciones de esos dos campos de pensamiento sobre los perjuicios, campos formados por las asociaciones medioambientales, por una parte, y por los científicos (ecólogos, 
médicos y biogeógrafos), por la otra, se reforzaron mutuamente; y permitieron, a unos, evocar los perjuicios que padecen, haciendo referencia a un posible peritaje científico, y a los otros, difundir entre el público los perjuicios y riesgos que este último no puede percibir directamente. En consecuencia, ya fue posible expresar legítimamente los perjuicios y los riesgos, pero unos y otros se mantuvieron en una dinámica compleja. En efecto, fueron entonces oponibles dos acciones cuyos contornos son vagos: la de la eliminación del origen de los perjuicios y los riesgos, y la de la simple compensación o la descontaminación; campos nuevos del mito progresista y campo de los grupos de presión económicos instruidos. Oposición que, por supuesto, la reducción del crecimiento y los empleos "verdes" no resumen (Challéat y Larceneux, 2011).

El juego de las partes interesadas en la conflictividad relacionada con la luz artificial es complejo; no obstante, se puede intentar una esquematización simple $-\mathrm{y}$, por ende, ¡simplista! - que permita comprender mejor las pocas grandes posiciones encontradas (véase la Figura 1). Como puede verse, en esos conflictos en torno a los llamados bienes públicos "puros" -porque no son excluibles ni apropiables (el agua, la salud, el paisaje, la noche, etcétera) -, el poder público sería con mucha frecuencia, al final, el actor hacia el cual se volverían todas las miradas y todas las esperanzas en materia de regulación.

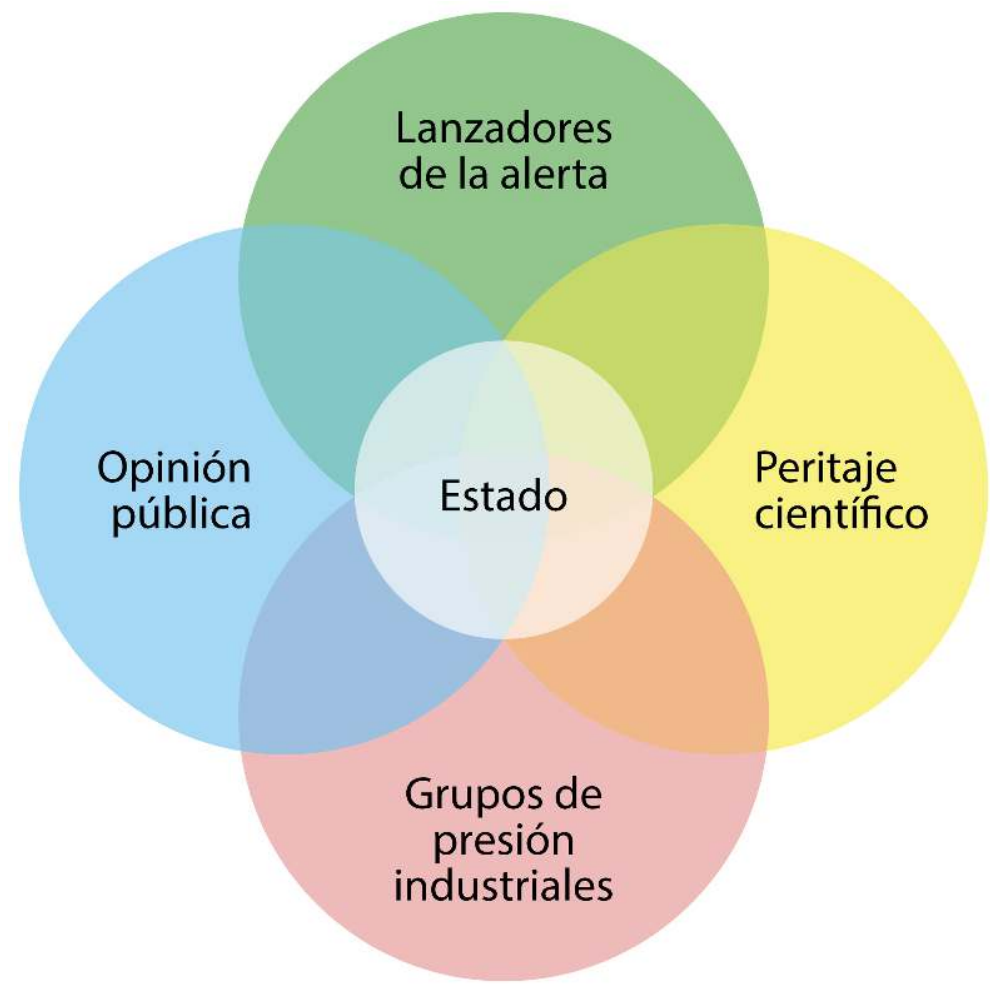

Figura 1. El juego de los actores en interacción con motivo de la fabricación de los problemas medioambientales y los conflictos que les son asociados.

En consecuencia, la percepción de un perjuicio no cae por su propio peso, independientemente de su acción real. El caso de la luz artificial es interesante porque está surgiendo y porque esa "contaminación" todavía se conoce muy poco; pero la misma dificultad se ha manifestado en el caso de todos los factores contaminantes actualmente reconocidos. Los peligros atribuidos al tabaco y, sobre todo, al tabaquismo pasivo son un buen ejemplo de ello. Como puede verse, lo que está en juego es poder concebir el perjuicio independientemente de las ventajas -individuales, sociales, políticas, industriales, etcétera- que podría procurar o, también, el inconsciente colectivo que 
acompaña a su objeto; asimismo, lo que está en juego es nuestra concepción de los valores que se encuentran en tela de juicio, con mayor motivo, puesto que los daños se ejercen sobre la "naturaleza" - jconcepto plástico por excelencia! - o el "medio ambiente". Hacer de la luz artificial un "objeto de perjuicio" y, por lo tanto, recíprocamente, de la noche un "objeto de salvaguarda" requiere la descentralización de la mirada, pasando del antropocentrismo hecho de racionalidad técnica a la valorización de los "objetos de naturaleza".

14 Valorización: el término podría remitirnos a los procesos económicos de evaluación de los daños, pero, antes, desde la perspectiva del análisis de la inclusión de ese nuevo problema medioambiental en la agenda política, debemos recurrir a los textos de la ética del medio ambiente (en el sentido de corriente filosófica) porque la protección de la noche frente al territorio de la luz se construye lentamente, desde hace ya muchas decenas de años, por medio de un proceso de valorización localizada del objeto "cielo negro estrellado" y, después, mediante la puesta en relieve del valor intrínseco de la negrura (Challéat, 2012).

\section{La puesta en tela de juicio de los valores éticos de la naturaleza y de su protección}

15 Como se ha visto, las nociones de fatalidad o necesidad, al igual que la de libertad individual absoluta, comprometen toda percepción de los perjuicios, los que, en el mejor de los casos, son percibidos de manera incidental como daños colaterales. La cuestión puede extenderse a la concepción de la naturaleza -y, por ende, del funcionamiento de los ecosistemas- y a la percepción de los riesgos. En efecto, así debió pensarse en el siglo XVII tanto el funcionamiento físico como el biológico del mundo, de la naturaleza, liberándose tanto del plan divino como de las causas finales y pensando en el perjuicio a partir de una interpretación científica: hacer a un lado la teología no se hizo sin obstáculos ni evoluciones erráticas y lo que permitió que el perjuicio surgiera de manera, no conceptual, sino tangible, localizada, fue claramente todo un proceso de construcción y valorización de lo que se veía amenazado, destruido, contaminado, etcétera (Challéat y Larceneux op. cit). 


\section{Nous savons que notre point de vue surl'Univers peut- être préservé. Nous savons que nous pouvons avoir des lumières nocturnes plus efficaces. La pollution lumineuse est un grand enjeu d'environnement. La Terre est importante. C'est ici que nous vivons et nous vivons dans l'UNIVERS. Faisons le pour les généra- tions futures !}

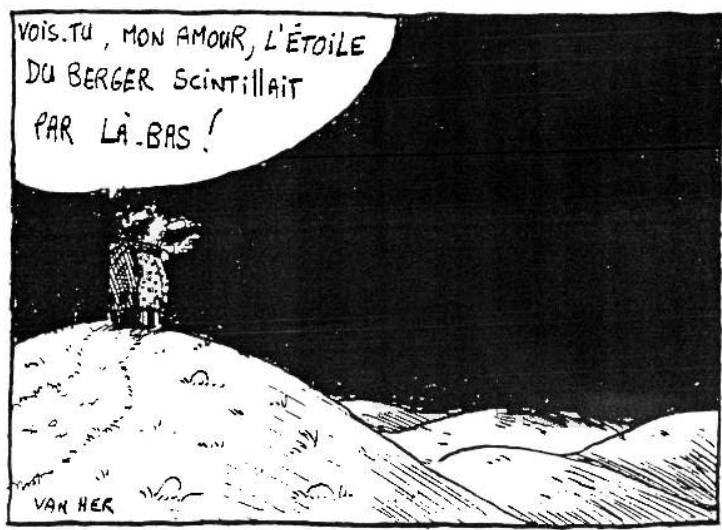

Ilustración extraída del dosier: La pollution lumineuse - Un véritable gâchis énergétique. Pourquoi ne peut-on pas admirer la voûte céleste depuis nos villes? Des solutions existent pourtant [La polución luminosa - Un verdadero derroche energético. ¿Por qué no podemos admirar la bóveda celeste desde nuestras ciudades? Existen soluciones, sin embargo], editado por la Fédération d'Astronomie Populaire Amateur du Midi. Finales de la década de 1980. Archivo del Colectivo RENOIR.

["Nosotros sabemos que nuestro punto de observación hacia el Universo puede ser preservado. Nosotros sabemos que podemos tener luces nocturnas más eficaces. La polución luminosa significa un gran desafío para el medio ambiente. La Tierra es importante. Aquí es donde vivimos y vivimos en el UNIVERSO. ¡Hagámoslo por las futuras generaciones!"]

Cartografía de la polución luminosa.

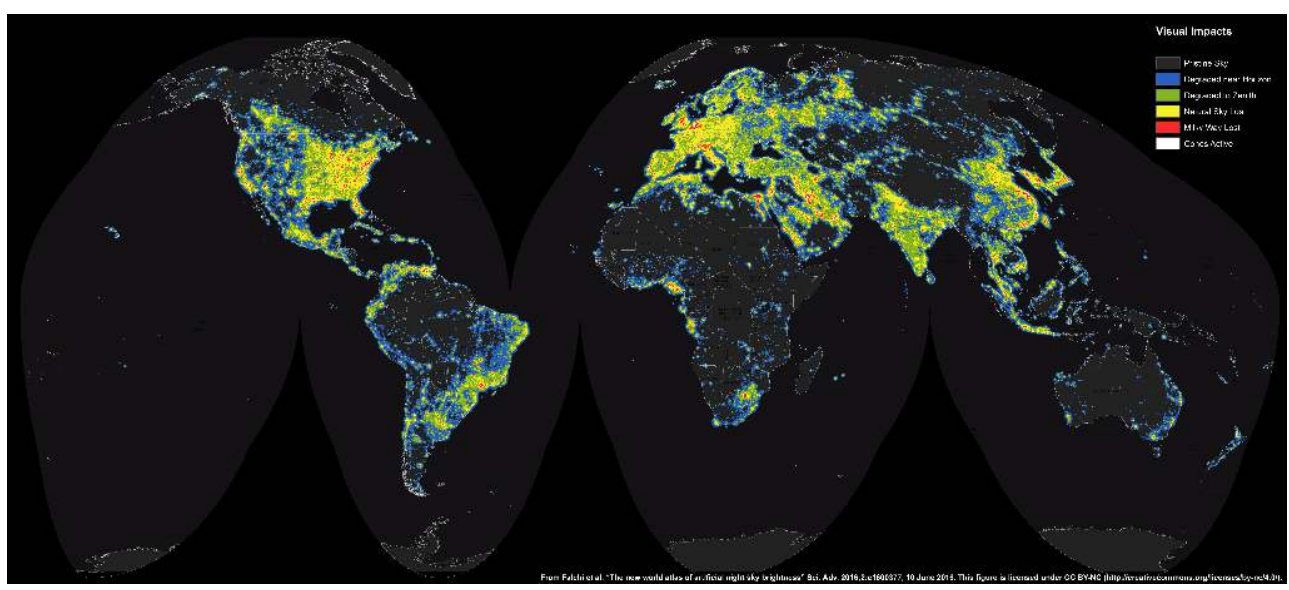

Cartografía de la calidad del cielo estrellado extraída de The new world atlas of artificial night sky brightness, publicado por Fabio Falchi et al. (2016) en Science Advances, Vol. 2, no. 6. DOI: 10.1126/ sciadv. 1600377 
Cuando los astrónomos concibieron la visión de la Vía Láctea como patrón de la intensidad de las molestias y la contaminación que la iluminación causaba, ${ }^{3}$ establecieron el valor de las zonas no contaminadas por la luz artificial y cubiertas por un cielo negro estrellado. Al final, ese patrón fue el que, en conjunto, adquirió la condición de objeto de la naturaleza dotado de un valor en el sentido entendido por la ética del medio ambiente y, por ende, la condición de objeto digno de consideración por sí mismo, fuera de toda consideración utilitarista y progresista en cuanto a la luz artificial y, por lo tanto, digno de ser protegido. Para los astrónomos, el punto de partida de su lucha fue suscitar el interés por el cielo negro estrellado. De acuerdo con Rolston: "El simple hecho de interesarse por algo constituye ipso facto un valor. Así, un objeto que, sin ello, sería un objeto sin valor puede llegar a tener un valor intrínseco" (Rolston, 1994: 13-30). En realidad, parece ser que, en lo concerniente al cielo nocturno, se está más cerca de un valor extrínseco no instrumental que de un verdadero valor intrínseco: únicamente se atribuye valor al cielo nocturno por sus aportaciones culturales, religiosas, científicas, contemplativas, etcétera; aportaciones claramente proyectadas por el hombre; y, ipor supuesto!, ¿aun cuando el valor de un sitio geográfico con un cielo extraordinariamente negro siga siendo un valor no instrumental, puesto que es el soporte de una promoción (un valor para el hombre)? (Poméon y Challéat, 2016).

El objeto cielo nocturno estrellado sería superado cuando, a partir de los años de 1990, los ecólogos y médicos llegaron a subrayar lo negro (la ausencia natural de luz) como un objeto, elemento o estado que forma parte directamente de todo biotopo y, por lo tanto, de la biocenosis o de la calidad sanitaria del medio ambiente. ${ }^{4}$ Por una preocupación de esquematización conceptual, se puede inferir las dos grandes posiciones éticas siguientes: para el ecólogo, el valor ya no se relaciona únicamente con el hombre; sin duda alguna, es instrumental, porque sirve a la biocenosis, pero claramente intrínseco, no decidido por el hombre, como el valor del agua, del aire y "de cada una de las substancias fundamentales de la biosfera" (Ferret, 2011). Para el médico, el valor de lo negro se relaciona completamente con el hombre y, en ese caso, también es instrumental (porque sirve directamente a la calidad sanitaria del medio ambiente), pero no es el trabajo (físico o intelectual) del hombre el que lo valoriza, por lo que, también en ese caso, lo negro tiene un valor intrínseco.

Se podría dedicar páginas enteras a discutir sobre los valores atribuidos a los objetos medioambientales "negro" y "cielo nocturno estrellado", así como sobre su relatividad. Si se ha abordado esta espinosa cuestión, fue porque las respuestas dadas reflejan claramente las diferentes posiciones de los actores en conflicto desde hace varios años. Para los defensores de la "nocturnidad", el negro es portador de un valor intrínseco instrumental: la vida necesita la alternancia natural del día y la noche, sin la cual, la erosión de la biodiversidad es ineluctable. En el caso de sus contradictores, lo más que hacen es conceder que la noche tiene un valor extrínseco instrumental, el cual sirve a los astrónomos y a la calidad sanitaria del medio ambiente. Esa oposición también se encuentra en los debates de las políticas destinadas al medio ambiente, sobre todo en lo concerniente al problema crucial de la evaluación de los daños que es necesario reparar. Según unos, la evaluación se puede llevar a cabo gracias a las herramientas de la economía (evaluación monetaria, precios hedonistas, consentimiento en pagar, etcétera), mientras que, para los otros, es simplemente imposible ("iLa noche es la vida!" y "ila vida no tiene precio!": el valor intrínseco es, de facto, un valor superior que lleva a un interés común superior). Para los primeros, a los que se podría calificar de 'tecnicistas', la 
compensación (aunque sería necesario ser capaces de cuantificar, incluso antes, conocer para reconocerlo- el perjuicio) o la descontaminación en un plazo más o menos largo por medio de la tecnociencia serán las soluciones. Para los segundos, sustentadores del medioambientalismo y el decrecimiento, se tratará de llamar a "erradicar el mal desde la raíz", para salvaguardar el valor del objeto de naturaleza, notablemente haciendo presión para que se lleve a cabo la creación de espacios protegidos, ya sean de reservación (conservación de su estado, lo que puede llevar al inmovilismo), de restauración (retorno a un estado anterior, a menudo imaginado, antes bien que realmente conocido, en ocasiones idealizado, incluso mitificado) o, más a menudo, intermedios, equipados por la gestión medioambiental del territorio y sus fronteras administrativas, sus "zonas núcleo", sus "zonas tapón".

En Francia, la inclusión en la agenda política de la problemática de los perjuicios y la contaminación de la iluminación artificial proporciona a las asociaciones medioambientales la ocasión para hacer que el debate se deslice hacia una controversia socio-técnica de la que uno de los problemas es "establecer una frontera clara y ampliamente aceptada entre lo que se considera como indiscutiblemente técnico y lo que se reconoce como indiscutiblemente social [...]. Reconocer su dimensión social [a lo que se podría añadir 'medioambiental'] significa dar una nueva oportunidad [a un expediente] para que se discuta en la arena política" (Callon, Lascoumes y Barthe, 2001) . Lo interesante en ese caso es la relación de unos y otros con el conocimiento científico actualmente imperfecto en materia de efectos sanitarios, por ejemplo-. Para los medioambientalistas, las pruebas científicas acumuladas por los ecólogos y los médicos bastan para tomar una decisión (principio de precaución), mientras que, para los tecnicistas, ese mismo saber científico deja todavía un lugar demasiado importante a la incertidumbre como para que pueda fundamentar esa misma decisión.

Ese uso estratégico del "estado actual del saber científico" debe ser reemplazado en las pruebas que tienen lugar con ocasión de los procesos legislativos y de normalización, pruebas que, aun cuando tengan lugar en escenas diferentes, son estructuradas por el antagonismo entre los tecnicistas y los medioambientalistas: dado que esas pruebas acercan o alejan a los actores de la controversia, determinan lo que la ley y la norma deben tomar en cuenta o no. Para los tecnicistas, lo que es mensurable es verificable, para los ambientalistas, es lo que podría ocurrir.

21 En consecuencia, su acercamiento tiene lugar en lo referente a la economía de la energía en todos los espacios; y, en la reciente Ley Grenelle 2, desembocó en una nueva zonificación (los sitios de observación astronómica, establecidos en espacios de baja densidad demográfica y de poca actividad) y en la toma en consideración de las molestias luminosas en las zonas medioambientales existentes (las reservas y los parques naturales, los sitios clasificados, etcétera). En sentido inverso, su alejamiento es observable en los espacios de alta densidad demográfica y de mucha actividad en los que el intrincamiento de los problemas sanitarios, medioambientales y económicos es más marcado. Al final, la temática sanitaria será sin duda alguna la que desempeñará la función del arbitraje: ya sea que se reconozca la peligrosidad de la iluminación para la salud humana, en cuyo caso lo que deberá ser protegido contra los perjuicios luminosos y los intereses económicos serán las zonas de alta densidad demográfica y sus habitantes; o ya sea que la peligrosidad de la iluminación no se revele con certidumbre y los intereses económicos se manifiesten más directamente. Con todo, independientemente de la salvaguarda de la noche contra la iluminación artificial, lo que entra en contradicción -hasta en sus modos de producción- 
con las reivindicaciones cívicas de orden medioambiental, que suponen aceptar la amplificación y la pluralidad del peritaje, es, con toda claridad, la normalización industrial y comercial (Challéat y Lapostolle, 2014).

\section{Conclusión}

¿Cómo pensar el perjuicio y el riesgo? ¿Cómo hacer de ellos un elemento autónomo, independiente de los beneficios que procura el desarrollo económico? Se comprende que, en un contexto "progresista", el pensamiento "negativo", a contracorriente, es difícilmente posible y audible: ¿cómo puede ser que el hecho mismo de concebir la luz que porta en sí el inconsciente colectivo de la vida, de la fuerza del Bien contra el Mal o incluso de la técnica- pueda ser un elemento perjudicial? Por consiguiente, es todo un proceso conflictivo lo que podrá hacer surgir ese pensamiento, en primer lugar, por medio de la descentralización intelectual, otra manera de percibir y concebir a partir de puntos de vista nuevos, irreductibles a la visión de lo divino o del progreso. En realidad, ello requiere pensar de otra manera o " más allá" en el terreno de las ideas, a partir de un campo conceptual que deberá estructurarse progresivamente, como fue estructurada por el astrónomo, el ecólogo y también el médico, la idea de la luz artificial como contaminante y, por lo tanto, de lo negro como recurso natural. Ese nuevo pensamiento, forzosamente radical, puesto que se encuentra en oposición a la "corriente predominante", no se puede comparar con el primero -el del "progreso" -, porque sus pautas de validez pertenecen a otro campo cognitivo y semántico y sus modos de representación de lo real siguen otros caminos.

Como ya se ha visto, los debates en torno al cielo nocturno estrellado, a la noche negra que lo hace visible y, después, más generalmente, en torno a lo negro como elemento constitutivo de todo biotopo y, por lo tanto, soporte de la biocenosis en conjunto y de la calidad sanitaria del medio ambiente nos remiten a ciertos conceptos y mecanismos utilizados y observados en muchos otros conflictos medioambientales relativos a los perjuicios o a los riesgos (ya sea que se piense únicamente en los debates en torno al cambio climatológico, el uso civil o militar de la energía nuclear, las ondas electromagnéticas, la contaminación medicamentosa, etcétera). ¿Es necesario aprehender el problema (y, por lo tanto, aportarle soluciones) de manera específica y local mediante la creación, por ejemplo, de Reservas Internacionales de Cielo Estrellado en torno a algunos observatorios astronómicos o algunos grandes sitios ya relativamente protegidos?; o, bien, ¿es necesario pasar a la toma en consideración más genérica y global, considerando el medio ambiente nocturno en conjunto, con sus múltiples facetas (sociales, ecológicas y sanitarias) y sus múltiples territorios, incluidos los urbanos? ¿Es necesario esperar que se refrene ese nuevo perjuicio por medio de nuevas técnicas o tecnologías (iluminación mediante leds, detectores de presencia, alumbrado de las calles a pedido por medio de los teléfonos portátiles, potenciómetros para variar la intensidad de la luz, etcétera)?, o bien, ¿es necesario emprender el camino del decrecimiento?

En esos debates, las nociones subyacentes de la ética medioambiental plantean sus interrogantes a escalas temporales difícilmente aprehendibles ("las generaciones futuras") cuando se piensa en la complejidad que define los sistemas -humanos o no- que están en juego; más aún, la reflexión exige que se tome en consideración espacios y territorios de tamaños variados, lo cual incrementa de facto la escala espacial de los problemas planteados... pero también ¡las soluciones que es necesario aportar! Del nicho 
ecológico del "gran pavón nocturno" [saturnia pyri] al ecosistema del ave migratoria, del conjunto autorregulado de los ecosistemas a la totalidad de la biosfera, de la persona que duerme en una pieza iluminada por una luz individual a los eventuales impactos sanitarios sobre el conjunto de la población urbana, el, los espacios son también complejos $\mathrm{y}$, por añadidura, imbricados. A esas escalas espacio-temporales de los perjuicios y los riesgos, no corresponden las escalas espacio-temporales de los beneficios extraídos de las actividades perjudiciales o contaminantes, lo cual muestra con claridad la recurrencia de los conflictos sobre la cuestión (Challéat y Larceneux, op. cit).

Finalmente, ante las respuestas muy a menudo técnicas aportadas a las cuestiones de los riesgos, los perjuicios y la contaminación (innovación tecnológica, normas de las que ya no se sabe muy bien si son de aplicación obligatoria o si se puede considerarlas como recomendaciones, pliegos de cláusulas técnicas particulares, etcétera) la inclusión en la agenda política de la protección del cielo nocturno y, más ampliamente, de la noche, remite a una interrogante muy importante: ¿cómo se fabrica el campo de lo que llamamos "los problemas medioambientales"? Dicho en otras palabras: según nuestra consideración en un momento y un espacio dados, ¿qué es un problema medioambiental y qué no lo es? Dejemos la temática del cielo nocturno poniendo de relieve que, en este caso, el conceptooxímoron del "desarrollo sostenible", de contornos vagos y ambiguos, proporcione algunas respuestas... jde una obscura claridad! Asimismo -para terminar con un tono provocador- lo que se puede poner legítimamente en tela de juicio son las políticas de reservación y, también, de restauración de "la naturaleza" aplicadas por los seres humanos, dado que también pueden llevar, ora al artefacto tecnocientífico, ora al conservadurismo ingénuo (Katz, 1992:231-241)

Entre, por una parte, la voluntad de reducir la naturaleza a un inmenso campo de experimentación y transformarla en un artefacto que produce las riquezas naturales $\mathrm{y}$, por otra parte, la voluntad de conservarla en el estado en que la encontramos o la soñamos en un pasado idealizado pero mítico, todas las opciones son posibles. Entre el mito cientificista y el romanticismo idealista, entre la búsqueda de las riquezas materiales producidas y las de los valores inmateriales de la Naturaleza o de la Cultura, ¿puede haber transigencia? Entre el cazador y el lobo, ¿quién es el depredador más legítimo (Larceneux, 2005:219-247).

Independientemente de todo lo anterior, la formación - ¿la fabricación?- de un patrimonio "natural", como, por ejemplo, el propuesto con ocasión del establecimiento de la Reserva Internacional de Cielo Estrellado, plantea el problema de la relación entre las cuestiones medioambientales y otras formas de patrimonio, ya sea que se piense únicamente en el patrimonio cultural, social, industrial e incluso económico. Pueden entonces surgir conflictos a diferentes escalas, entre la preservación o la recreación impuesta con mucha frecuencia o resentida como tal- de uno y el mantenimiento de los otros; pero, en algunas ocasiones, esos conflictos pueden llevar a algunas formas de cooperación entre las actividades en un mismo territorio. Esa sinergia supone la convergencia de saberes específicos cuando se lleva a cabo el arbitraje entre la cuestión medioambiental y otras formas, a menudo ya existentes, de patrimonio y conocimiento. El porvenir de la muy reciente Reserva Internacional de Cielo Estrellado creada en los alrededores del observatorio astronómico del Pic du Midi de Bigorre, en los Pirineos, por ejemplo, nos dirá si y cómo los territorios pueden apropiarse la cuestión de la valorización de la nocturnidad e integrarla durablemente en su gestión económica, cultural y turística (Bénos et al., 2016). 


\section{BIBLIOGRAFÍA}

Afeissa, H.-S., 2007, Éthique de l'environnement. Nature, valeur, respect, Vrin, Paris.

Barjonet, P., M. Gezentsvey y C. Mores, 2010, "Perception des risques et choix du mode de transport. Approche conceptuelle et modélisation prédictive”, Flux, vol. 3, núm. 81, pp. 19-32.

Bénos R., Challéat S., Lapostolle D., Dupuy P.-O., Poméon T., Milian J. y Girard F., 2016, Protéger la nuit d'un haut lieu touristique de montagne. La Réserve Internationale de Ciel Étoilé du Pic du Midi de Bigorre comme nouvelle ressource territoriale. En Delaplace M. et Gravari-Barbas M., (Eds.), Nouveaux territoires touristiques, Presses de l’Université du Québec, Québec.

Bortle, John E., 2001, "Introducing the Bortle Dark-Sky Scale - How dark is your sky, really? Find out how to rate your observing site", Sky \& Telescope, vol. 101, num. 2, p. 126.

Cabantous, A., 2009, Histoire de la nuit. XVII ${ }^{e}-\mathrm{XVIII}{ }^{e}$ siècle, Fayard, Paris.

Callon, M., P. Lascoumes y Y. Barthe, 2001, Agir dans un monde incertain. Essai sur la démocratie technique, Le Seuil, collection La Couleur des idées, Paris.

Challéat, Samuel, 2010, «Sauver la Nuit » - Empreinte lumineuse, urbanisme et gouvernance des territoires, thèse de doctorat en géographie, Université de Bourgogne.

Challéat, Samuel, 2012, La mise en débats des territoires de la lumière, Actes du congrès du GIS Démocratie \& Participation, du 18 au 21 octobre 2011, EHESS, Paris.

Challéat, Samuel, y A. Larceneux, 2011, Pour une approche cognitive et multiscalaire des conflits environnementaux, $48^{e}$ colloque de l'ASRDLF, du 7 au 9 de juillet 2011, Schœlcher, Martinica.

Challéat S. y Lapostolle D., 2014, (Ré)concilier éclairage urbain et environnement nocturne : les enjeux d'une controverse sociotechnique, Natures Sciences Sociétés, 22(4), 317-328. En línea: http:// www.nss-journal.org/articles/nss/abs/2014/04/nss140045/nss140045.html

Delattre, S., 2004, Les Douze Heures noires - La Nuit à Paris au XIX siècle, Albin Michel, collection Bibliothèque de l'Évolution de L'Humanité.

Falchi, Fabio, Cinzano1, Pierantonio, Duriscoe, Dan, Kyba, Christopher C. M., Elvidge, Christopher D., Baugh, Kimberly, Portnov, Boris A., Rybnikova, Nataliya A., y Furgoni, Riccardo, 2016, The new world atlas of artificial night sky brightness, en Science Advances, Vol. 2, no. 6. DOI: 10.1126/ sciadv.1600377.

Ferret, S., 2011, Deepwater Horizon - Éthique de la nature et philosophie de la crise écologique, Le Seuil, collection L'Ordre philosophique, Paris.

Hirschman, A. O., 1970, Exit, Voice, and Loyalty: Responses to Decline in Firms, Organizations, and States, Harvard University Press, Cambridge.

Katz, E., 1992, "The Big Lie: Human Restoration of Nature" [conferencia pronunciada en junio de 1990 en ocasión del coloquio dedicado a la Philosophie morale dans le domaine public en la Universidad de Columbia], Research in Philosophy and Technology, vol. 12, pp. 231-241.

Koslofsky, C., 2011, Evening's Empire - A History of the Night in Early Modern Europe, Cambridge University Press, Cambridge. 
Larceneux A., 2005, Les Conflits d'interprétation dans les approches environnementales : propositions pour un dépassement, Actes des Premières journées scientifiques de l'économie de l'environnement : les stratégies des acteurs, Algérie, 1 y 2 de octobre 2005, Alger, pp. 219-247.

Mons, A., 2000, La communication lumière de la ville. Un devenir-image des lieux, Médiation et information, núms. 12-13.

Moscovici, S., 1984, “The Phenomenon of Social Representations”, en R. Farr y S. Moscovici (coords.), Social Representations, Cambridge University Press, Cambridge, pp. 3-69.

Narisada, K., y D. Schreuder, 2004, Light Pollution Handbook, Astrophysics and Space Science Library, Berlin/New York/London, Springer.

Poméon T. y Challéat S., 2016, "And What do You do With Five-hundred Millions of Stars?" Reflections on the Asessment of the Night and the Starry Sky through their Protection in the Territories. En Hoskins G. y Saville S., (Eds.), Locating Value: Theory, Application and Critique, Taylor \& Francis Books, UK.

Rich, C., y T. Longcore (coords.), 2006, Ecological Consequences of Artificial Night Lighting, Island Press, Washington.

Rolston, H., 1994, "Value in Nature and the Nature of Value" [conferencia pronunciada ante la Royal Society of Philosophy en la Universidad de Gales, Cardiff, 18 al 21 de junio de 1993], en R. Attfield y A. Belsey (coords.), Philosophy and Natural Environment, Cambridge University Press, Cambridge, 1994, pp. 13-30.

Thériault, M., R. Landry, J. Rouffignat, P. Levallois, C. Chiasson, S. Tessier, M. Girard y C. Previl, 2000, La perception des nuisances environnementales par les résidents de Portneuf, en R. Landry y P. Levallois, coords., Agriculture intensive et écosystèmes régionaux: Du diagnostic aux interventions, Presses de l'Université Laval, Québec.

\section{NOTAS}

1. En 1995, el Comité Nacional de Protección del Cielo Nocturno (CNPCN), que vio la luz del día en 1994 en el seno de la Sociedad Astronómica de Francia, emitió una carta intitulada “¡Salvemos la noche!" -versión actualizada de la "Carta para la preservación del medio ambiente nocturno" (1993)-, que estipula en su preámbulo que "[Los signatarios] Comprueban que, desde hace mil millones de años, la vida en la Tierra estaba regida por la alternancia del día y la noche; pero, en unas cuantas decenas de años, el equilibrio natural fue roto por la proliferación anárquica de una iluminación urbana demasiado agresiva y mal distribuida. En consecuencia, es urgente poner un alto a los perjuicios resultantes [...]".

2. En este caso, como ocurre con mucha frecuencia cuando surge la percepción de una molestia más o menos grande entre cierta categoría de actores, es necesario subrayar la importante función de la observación medioambiental directa (véase Thériault, Landry, Rouffignat et al., 2000), y es posible comparar los mecanismos encontrados en la movilización de los astrónomos contra los perjuicios luminosos con los ya encontrados en el surgimiento de numerosos riesgos (véase P. Barjonet, M. Gezentsvey y C. Mores, 2010: 19-32).

3. La escala de cuantificación de la contaminación luminosa más popular entre los astrónomos aficionados es la "escala Bortle", publicada en 2001 por John E. Bortle en la revista de divulgación Sky \& Telescope (véase Bortle 2001: 126). Dicha escala está compuesta de nueve clases y se basa en la visibilidad a simple vista de diferentes fenómenos (la luz zodiacal, por ejemplo) u objetos astronómicos, entre ellos, la Vía Láctea; un cielo de clase 7, por ejemplo, que corresponde a la "transición entre los suburbios y la ciudad", es descrito de la siguiente manera: "El cielo muestra 
un color ligeramente azulado, teñido de anaranjado y marrón. La Vía Láctea es completamente invisible [...]", mientras que un cielo de clase 1 , que corresponde a "un excelente cielo negro", es un "cielo exento de todo fenómeno luminoso artificial. La luz zodiacal, el resplandor antisolar, la banda zodiacal y toda la Vía Láctea son perfectamente discernibles [...]". El artículo se encuentra disponible en línea en la siguiente dirección: https://www.nps.gov/subjects/nightskies/upload/ BortleDarkskyScale-2.pdf

4. Los ecólogos ponen como ejemplo los costos medioambientales de la iluminación y muestran ecosistemas marcadamente perturbados por la luz artificial; y los biólogos observan numerosos efectos sobre la fauna en varias escalas, efectos que dependen esencialmente, a pequeña escala, de los mecanismos de atracción y repulsión de las fuentes luminosas, así como de las perturbaciones, a más grande escala, de ciertas especies desorientadas por los halos luminosos. Asimismo, han sido observados numerosos trastornos del comportamiento en las comunidades y los ecosistemas a escalas de tiempo variables. Desde el punto de vista de la salud humana, las investigaciones médicas muestran que la alternancia natural del día y la noche es el primer marcador del tiempo del reloj interno de los seres humanos: ese sincronizador exógeno regula el ritmo circadiano de secreción de varias hormonas, sobre todo de la melatonina: la falta de sincronización de su secreción puede generar tensión, fatiga, degradación de la calidad del sueño, irritabilidad o trastornos del apetito. La buena calidad del ritmo circadiano de secreción de la melatonina, en fin, podría frenar la aparición de ciertos tipos de cáncer. Para consultar una reseña bibliográfica de los efectos e impactos sobre el medio ambiente y la salud de la luz artificial, se remite al lector a las tres referencias siguientes: Samuel Challéat, "Sauver la Nuit"..., op. cit.; K. Narisada y D. Schreuder, 2004; y Rich, 2006.

5. Véase también Afeissa, 2007.

\section{AUTORES}

\section{SAMUEL CHALLÉAT}

Samuel Challéat es geógrafo y planificador territorial, investigador contractual en el UMR CNRS 5193 LISST-Dynamiques Rurales (Universidad de Toulouse 2-Jean Jaurès). Cofundador en 2013 del Colectivo de investigación RENOIR (Ressources Environnementales Nocturnes \& territOIRes) que también coordina; es el autor de la primera tesis de Doctorado que se ocupa de la problemática emergente sobre las molestias y la contaminación lumínicas ( Sauver la nuit ». Empreinte lumineuse, urbanisme et gouvernance des territoires), presentada en 2010. Dicha tesis explora las mutaciones en la producción y la gobernanza de la iluminación urbana ante las nuevas preocupaciones medioambientales y económicas. Se dedica especialmente a analizar el papel de los actores que causan las molestias y la polución lumínicas como un problema público para llevarlo a la agenda política. Sus trabajos hoy se concentran en el análisis de las acciones colectivas territorializadas de protección y de valorización del cielo estrellado y del medio ambiente nocturno en relación con el referencial de la transición energética en varios campos diversificados (zonas rurales, montañas, espacios periurbanos y zonas protegidas). 